\title{
Pregnant woman with an intracranial meningioma - case report and review of the literature
}

\author{
Bogdan Constantin Dumitrescu ${ }^{1,2}$, Ligia Gabriela Tataranu ${ }^{2,3}$, \\ Mircea Radu Gorgan ${ }^{2,3}$ \\ ${ }^{1} \mathrm{PhD}$ student, University of Medicine and Pharmacy "Carol Davila”, Bucharest \\ ${ }^{2}$ Clinic of Neurosurgery, Emergency Clinical Hospital "Bagdasar Arseni”, Bucharest \\ ${ }^{3}$ University of Medicine and Pharmacy "Carol Davila", Bucharest
}

\begin{abstract}
It is about a 33-year-old female, with a 36 weeks uncomplicated pregnancy and with signs of increased intracranial pressure. Hours after admission and an obstetric evaluation, uterine contraction started and the patient was taken to the delivery room, where she presented a partial motor seizure on the left side with secondary generalization and urine emission. A caesarean section was performed without fetal or maternal complications. The urgent MRI gadolinium-enhanced brain scan revealed a 39/50/54 mm tumoral mass having an aspect of an anterior third falx cerebri meningioma. The patient was transferred to our neurosurgical department and afterwards surgery was performed with gross total removal of the tumoral mass. Histological examination revealed atypical meningioma with direct invasion into the adjacent brain parenchyma. A week later she was discharged from the hospital in good condition. One month after surgery, a contrastenhanced magnetic resonance imaging of the brain did not reveal any signs of tumor recurrence or residual tumor. Our recommendation is for postpartum surgery when is possible. Urgent neurosurgical interventions should be made in case of patients with malignant tumors, active hydrocephalus or benign intracranial tumor such as meningioma associated with signs of impending herniation, progressive neurological deficits.
\end{abstract}

\section{Introduction}

Intracranial tumors in pregnant women are rare, with an estimated incidence of about seven cases per 125.000 pregnancies. The first case of a pregnant woman with a brain tumor was described by Bernard in 1898. $(1,2,3,4,5)$

The occurrence of a meningioma during pregnancy is also rare, comparable with that in non-pregnant women in the same age group. $(1,2,3,4)$

Particular for meningiomas is the expression of hormone receptors. These tumors response to the increased level of serum progesterone during the second half of pregnancy by accelerating its growth. Tumor 
enlarges by fluid retention, enhanced vascularity and therefore patients may become symptomatic. (1)

The clinical presentation with signs and symptoms of elevated intracranial pressure, focal seizures, lateralising neurological deficits, abnormal funduscopic examination suggests the diagnosis of an intracranial mass and should not be confused with hyperemesis gravidarium, eclampsia or puerperal psychosis. $(1,5)$

Magnetic resonance imaging of the brain is the method of choice for prompt diagnosis of meningioma during pregnancy. (1)

The management strategy for intracranial tumors during pregnancy should be individualised and include mainly surgery. Surgery during pregnancy associates a great risk for fetus and also for the mother. The general recommendation is for caesarean section as first surgery and then the neurosurgical intervention when the patient's neurological status and the gestational age allow. (9)

Urgent neurosurgical interventions should be made in case of patients with malignant tumors, active hydrocephalus or benign intracranial tumor such as meningioma associated with signs of impending herniation, progressive neurological deficits. (5)

Radiotherapy and mifepristone are recommended for unresectable or recurrent meningioma. (4)

\section{Case report}

A 33-year-old right-handed female, 36 weeks pregnant presented to her obstetrician with one month history of headache, 24 hours before admission she accused repeated vomiting. An obstetric evaluation revealed an uncomplicated pregnancy at 36 weeks. The ultrasound and the fetal monitoring was in normal ranges. Four hours from admission uterine contraction started and the patient was taken to delivery room, here she presented a partial motor seizure on the left side with secondary generalization and urine emission. After seizure the patient was drowsy, her mental status depressed progressively. She was urgent transfer to operation room and caesarean section was performed under general endotracheal anesthesia. The caesarean section went well without fetal or maternal complications.

After delivery the patient underwent imaging investigation. The urgent IRM scan of the brain with gadolinium revealed a 39/50/54 $\mathrm{mm}$ tumoral mass with the same density, which captured intense and homogeneous contrast. The tumor was plated on the anterior $1 / 3$ of the falx cerebri on the right side. Midline was shifted to the left side by about $14 \mathrm{~mm}$. The tumor was also accompanied by significant perilesional edema. (Figure 1)

In this stage the patient was transferred to our neurosurgical department. On admission the patient was afebrile with normal vital signs. The general examination was normal. The neurological exam revealed impaired consciousness, left-sided hemiparesis with a left-sided pronator drift. Reflexes were diminished on the left side and the Babinski sign was present on the left side. The funduscopic examination showed bilateral papillary edema.

Anticonvulsant and corticoid therapy was immediately started and the neurological status of the patient improved. 
DOI: 10.2478/romneu-2014-0067
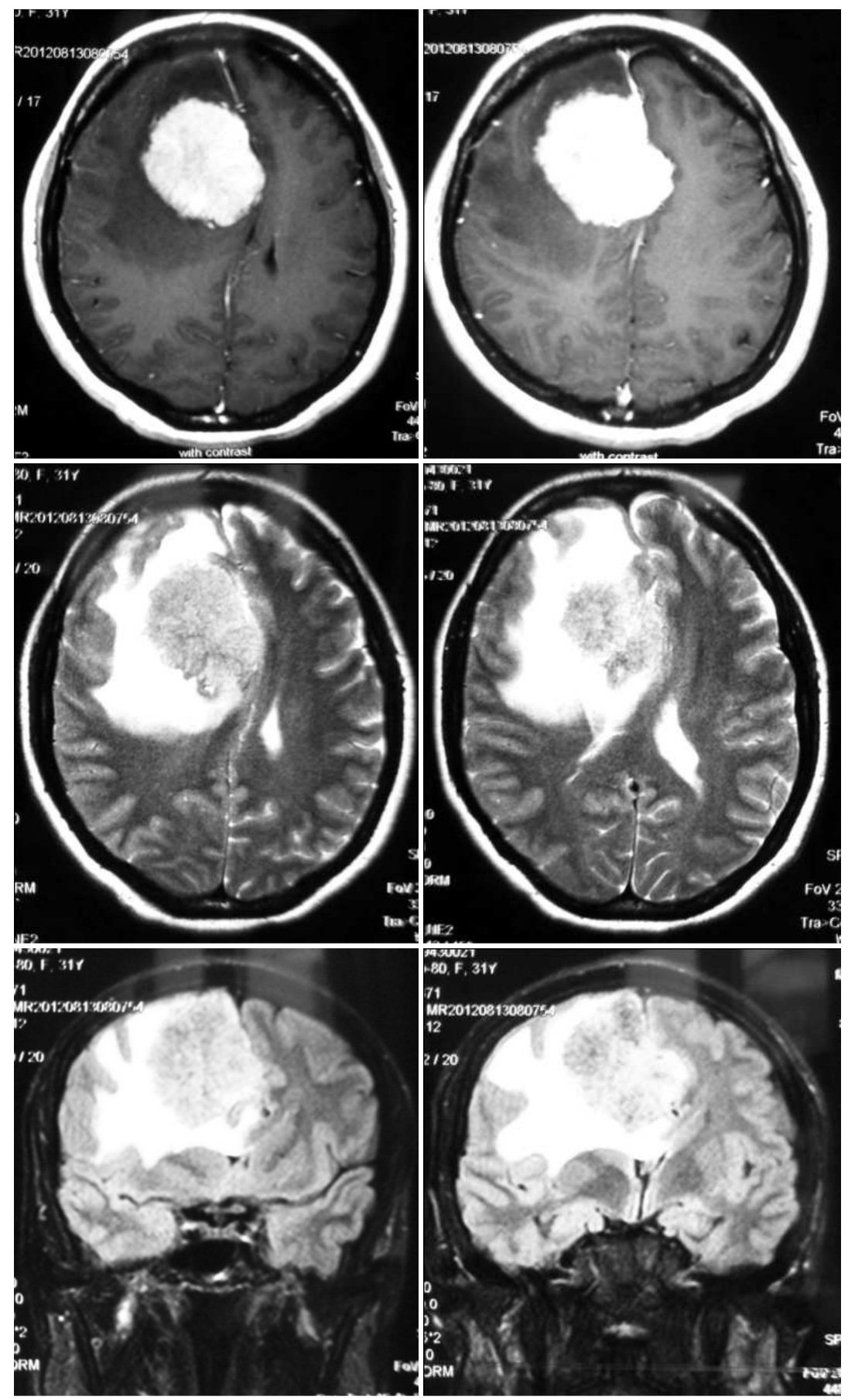

Figure 1 - Preoperative MRI. Well-defined, contrast enhancing, extraaxial tumor, attached to the anterior third of falx cerebri, with mass effect and surrounding edema 

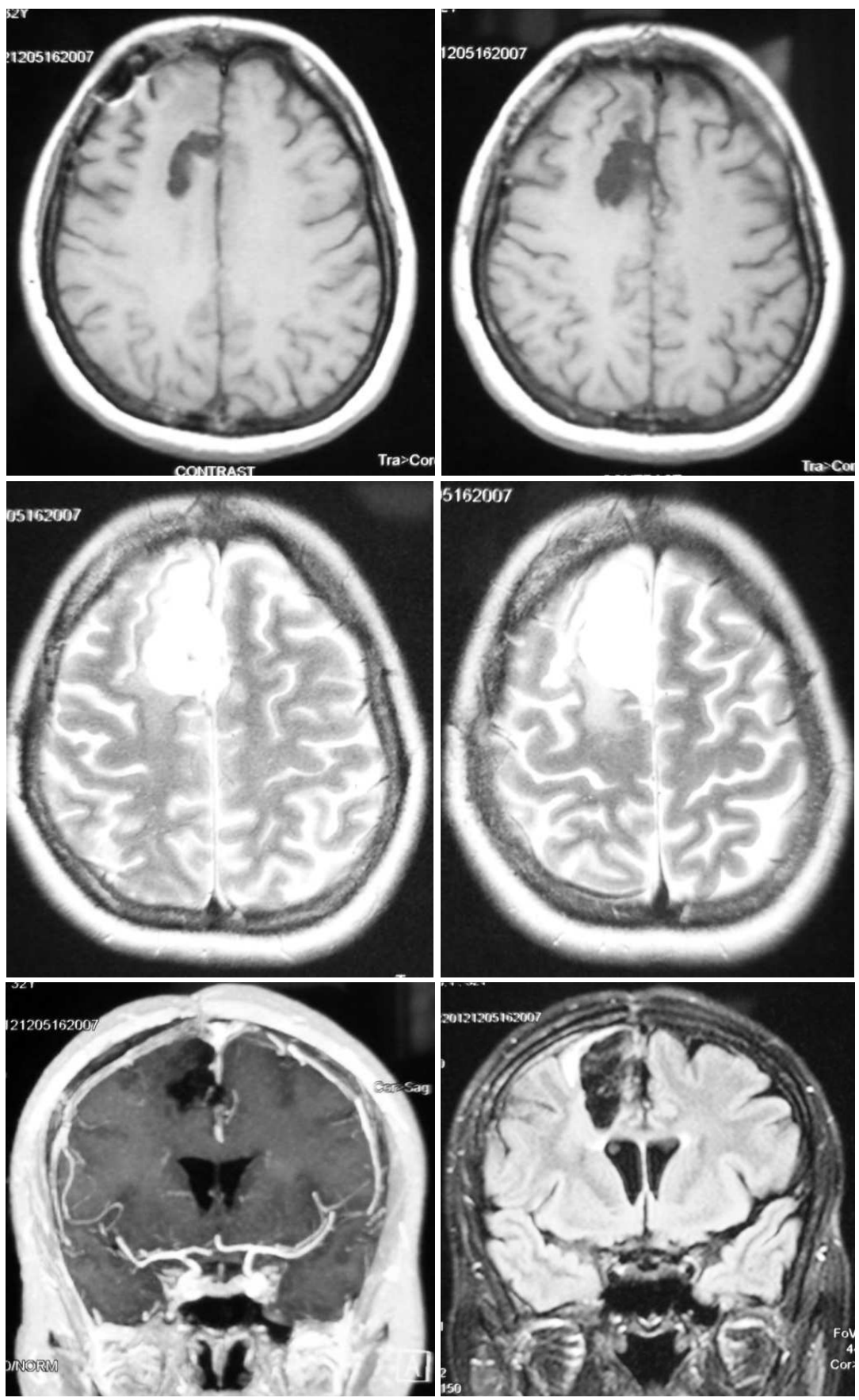

Figure 2 - Postoperative MRI. Complete tumor resection, no tumor recurrence 
DOI: 10.2478/romneu-2014-0067

After few days the neurosurgical operation was scheduled. Under general anesthesia a right frontal craniotomy was performed. The tumor was easily identified as a hypervascular mass adherent to the anterior $1 / 3$ of the falx. A peritumoral plane of dissection was developed and tumor was resected with coagulation of its falx adhesions.

Histological examination revealed atypical meningioma with direct invasion into the adjacent brain parenchyma.

After surgery patient was conscious, with minimal left hemiparesis and no seizure. She was discharged from the hospital 7 days later, in good condition.

Approximately one month after surgery, a contrast-enhanced magnetic resonance imaging of the head was performed; it does not reveal any signs of tumor recurrence or residual tumor. (Figure 2)

\section{Discussion}

Meningioma is the most common primary intracranial tumor in the adult population; it is a slow growing extra-axial brain neoplasm that arise from the arachnoid cells. This tumor accounts for $10-20 \%$ of all central nervous system neoplasms with an incidence among women of 3 in 100.000. The occurrence of a meningioma during pregnancy is rare because pregnant women tend to be young and healthy, so the general incidence of this pathology does not exceed that of nonpregnant women in the same age group. $(1,2,3,4)$

In 1898, Bernard described the first case of a pregnant woman with a brain tumor. In 1937, Hagedoorn presented a documented study about the effects of pregnancy on intracranial meningioma. $(5,6,7)$. The relationship between pregnancy and the rapid increase of neurologic symptoms in women with meningioma was first described by Cushing and Eisenhardt. $(8,9)$

The predominance of meningiomas in women and accelerated growth during luteal phase of menstrual cycle and during pregnancy is determined by the expression of progesterone and estrogen receptors. Swensen and Kirsch noted in their study from 2002 that meningiomas express estrogen receptors in $13 \%$ of cases and progesterone receptors in 69 $\%$ of cases. $(4,10)$

Meningiomas may also express hormone receptors not only for progesterone and estrogen but also for androgen, placenta growth factor andother exogenous hormones. $(1,11,12,13)$ These tumors response to increased level of serum progesterone in the second half of pregnancy by accelerated growth, so the tumor enlarges by fluid retention and enhanced vascularity. $(1,9,14)$

The acceleration of meningioma growth in the second or third trimesters of pregnancy may be determined also by the absence of Luteinising hormone and Follicle-stimulating hormone and by the falling level of Human chorionic gonadotropin in these stages of pregnancy. These observations were made in a vitro study on meningiomas by Boyle-Walesh, Shenkin and White in their paper published in 1995. (15)

The phenotypic profile of hormone receptor in meningioma has been classified into a high percentage of tumors displaying high level of progesterone receptor (PR); a smaller subgroup containing moderate concentrations of androgen receptor (AR); the level of estrogen receptor (ER) has been equivocal, ranging from low to virtually 
undetectable by immunohistochemistry. ( 9 , 16) In our days, Positron emission tomography helps us to measure in vivo the receptor density and occupancy in this type of tumor. (17)

The main signs and symptoms of intracranial meningioma are headache, vomiting and seizure, this clinical picture can be confused with hyperemesis gravidarium, puerperal psychosis in the first trimester of pregnancy or with eclampsia in late pregnancy. $(1,5)$ The presence of papillary edema, visual disturbances, focal seizure, and lateralizing neurological deficits should suggest an intracranial lesion.

The appearance or aggravation of signs and symptoms during pregnancy is determined by one of the following mechanisms: maternal metabolic changes causing fluid retention, vascular engorgement, and increased edema or positive hormone receptors on tumor cells with dramatic growth of the meningioma. The presence of progesterone receptors on tumor cells is correlated with worsening symptoms of meningioma. $(5,18,19)$

Therefore, in women in late pregnancy presenting with symptoms of increased intracranial pressure and focal signs or presenting with progressive worsening of neurological symptoms, an intracranial pathology should be considered and further imagistic investigations should be made to establish correct diagnosis.

Magnetic resonance imaging of the brain is the method of choice for prompt diagnosis of meningioma during pregnancy. We must note its limitations due to the inability to use contrast agents during pregnancy. The CT scan is not recommended because carries the risk of radiation damage. (1)
The management strategy for intracranial tumors during pregnancy should be made depending on patient's physical status, localization of the tumor, gestational age and other concomitant factors. Surgical excision is the treatment of choice for intracranial meningiomas but surgical intervention should be avoided during pregnancy when is possible because of the increased risk to both mother and fetus. Postpartum surgery of meningioma is recommended by most authors in the literature, with non-surgical treatment of the increased intracranial pressure until fetal lung maturity is reached. $(9,18)$ Others consider that non-invasive fetal monitoring such as fetal biophysical profit scoring system and umbilical artery Doppler velocimetry, and neuroanesthesia and microsurgical techniques permit a safe neurosurgical treatment of intracranial tumors during pregnancy. (5)

Our recommendation is for postpartum surgery when is possible. Urgent neurosurgical interventions should be made in case of patients with malignant tumors, active hydrocephalus or benign intracranial tumor such as meningioma associated with signs of impending herniation, progressive neurological deficits. (5)

Neurosurgery during pregnancy associates important risks for the mother. General endotracheal anesthesia is complicated by the upper airway mucosal edema, decreased functional residual capacity, venous engorgement, water retention, increased risk for reflux and aspiration, weight gain. In pregnancy the use of diuretics is not recommended so the neurosurgeon will confront with the lack of brain relaxation and that makes the operation more difficult. The surgery can also induce labor and it can be 
DOI: 10.2478/romneu-2014-0067

associated with important blood loss. (1, 20, 21, 22)

Brain surgery can also harm the fetus, it can cause stillbirth, birth defects (in the early stage of pregnancy), premature labor, premature delivery or fetal asphyxia. $(1,23)$

From another perspective, if the neurosurgery is not made, the tumor may enlarge, and it can cause seizure or even herniation. $(1,21,24)$

Complete surgical resection is the only definitive cure for meningiomas. Complete resection is determined by the tumor site. Mirimanoff et al, consider that total excision depends on the accessibility of the tumor $(28 \%$ for sphenoid wing and $96 \%$ for convexity meningiomas). High risk of recurrence is also associated with atypical or anaplastic meningioma and with a proliferative index greater than or equal to $20 \%$. $(25,26,27)$

Medical treatment before and after surgery includes corticosteroids, the usual dose is 2-4 $\mathrm{mg}$ of dexamethasone every 6 hour. Anticonvulsant medication is also needed, preferably monotherapy. The patients on anticonvulsant therapy should receive folic acid and supplemental vitamin-K1 three weeks before and during confinement to minimalize the risk of drug induced neural tube defects or blood dyscrasias. Manitol should be administrated only for acute emergency because sudden decrease in plasma volume might compromise uteroplacentar perfusion and put the fetus to risk. $(28,29,30)$

Mifepristone, hidroxyurea as antiprogesterones, temozolomide or radiotherapy can be used for unresectable benign meningioma, recurrent or malignant meningioma. Interferon as an angiostatic may also be considered. $(31,32,33)$

\section{Conclusions}

The management of a pregnant woman with an intracranial meningioma should be individualized and the final decision must be made on the intracranial pathology, gestational age and not least to be taken into account the patient's wishes. Pregnant women with repeated vomiting, nausea and headache should be examined with care and an intracranial pathology should be considered.

ACKNOWLEDGEMENT: This paper was co-financed from the European Social Fund, through the Sectorial Operational Programme Human Resources Development 2007-2013, project number POSDRU/159/1.5/S/138907 "Excellence in scientific interdisciplinary research, doctoral and postdoctoral, in the economic, social and medical fields EXCELIS", coordinator The Bucharest University of Economic Studies.

\section{Correspondence}

Bogdan Constantin Dumitrescu, address:

Emergency Clinical Hospital Bagdasar-Arseni, No. 10-12, Berceni Street, Sector 4, Bucharest; e-mail: dumitrescu.bog@gmail.com; tel.0745.696.966

\section{References}

1. Kasper EM, Hess PE, Silasi M, Lim KH, Gray J, Reddy H, Gilmore L, Kasper B. A pregnant female with a large intracranial mass: Reviewing the evidence to obtain management guidelines for intracranial meningiomas during pregnancy. Surg Neurol Int. 2010 Dec 25; 1:95

2. Sahu S, Lata I, Gupta D.Management of pregnant female with meningioma for craniotomy.J Neurosci Rural Pract. 2010 Jan;1(1):35-7

3. Rohringer M, Sutherland GR, Louw DF et al. Incidenceand clinicopathological features of meningioma. J Neurosurg 1989;71:665-72

4. Lucy D, Pratima M, Puspa K, Umakant S, Niharika P. Pregnancy with meningioma. J Obstet Gynecol India.2008 March/April; 58 (2): 156-158 
5. Kanaan I, Jallu A, Kanaan H. Management Strategy for Meningioma in Pregnancy: A Clinical Study. Skull Base. 2003 Nov;13(4):197-203

6. Bernard MH. Sarcome cerebral à evolution rapide au cours de la grossesse et pendant les suites des couches. Bull Soc d'Obst de Paris 1898;1:296-298

7. Hagedoorn A. The chiasmal syndrome and retrobulbar neuritis in pregnancy. Am J Ophthalmol 1937;20:690-699 8. Cushing $\mathrm{H}$, Eisenhardt L. Meningiomas. Their classification, regional behaviour, life history, and surgical end result. Springfield, Illinois: 1938.

9. Alluwimi I, Al-Anazi AR. Meningioma in pregnancy. Bahrain Madical Bulletin. 2004 June; 26 (2): 1-8.

10.Gabos S, Berkel J. Meta-analysis of progestin and estrogen receptors in human meningiomas. Neuroepidemiology 1992; 11:255-60.

11.Carroll RS, Zhang J, Black PM. Expression of estrogen receptors alpha and beta in human meningiomas. J Neurooncol 1999; 42: 109-16.

12.Donnini S, Machein MR, Pateu KH, Weich HA. Expression and localization of placenta growth factor and PIGF receptors in human meningiomas. J Pathol 1999; 189: 66-71.

13.Claus EB, Black PM, Bondy ML, Calvocoressi L, Schidkraut JM, Wiemels JL, et al. Exogenous hormone use and meningioma risk: What do we tell our patients? Cancer 2007; 110: 471-6.

14.Hatiboglu MA, Cosar M, Iplikcioglu AC, Ozcan D. Sex steroid and epidermal growth factor profile of giant meningiomas associated with pregnancy. Surg Neurol 2008; 69: 356-62.

15.Boyle-Walesh E, Shenkin A, White MC, et al. Effect of gylcoprotein and protein on human meningioma cell proliferation in vitro. J Endocrinol 1995;145:155-161.

16.Konstantinidou AE, Korkolopoulou P, Mahera H, et al. Hormone receptors in non-malignant meningiomas correlate with apoptosis, cell proliferation and recurrence-free survival. Histopathology 2003;43:280-1. 17.Moresco RM, Scheithauer BW, Lucignani G, et al. Oestrogen receptors in meningiomas: A correlative PET and immunohistochemical study. Nuclear Medicine Communications 1997; 18:606-15.

18.Benzel EC, Gelder FB. Correlation between sex hormone binding and peritumoral edema in intracranial meningiomas. Neurosurgery 1988;23:169-74.

19.Balki M, Manninen PH. Craniotomy for suprasellar meningioma in a 28 -week pregnant woman without fetal heart rate monitoring. Can J Anaesth. 2004; 51:573-6. 20.Finfer SR. Management of labour and delivery in patients with intracranial neoplasms. $\mathrm{Br} J$ Anaesth.1991;67:784-7.

21.Wang LP, Paech MJ. Neuroanesthesia for the pregnant woman. Anesth Analg 2008;107:193-200.

22.Chang L, Looi-Lyons L, Bartosik L, Tindal S. Anesthesia for cesarean section in two patients with brain tumours. Can J Anaesth 1999;46:61-5.

23.Goldberg M, Rappaport ZH. Neurosurgical, obstetric and endocrine aspects of meningioma during pregnancy. Isr J Med Sci. 1987;23:825-8.

24.Haas JF, Janisch W, Staneczek W. Newly diagnosed primary intracranial neoplasms in pregnant women: A population-based assessment. J Neurol Neurosurg Psychiatry. 1986;49:874-80.

25.Mirimanoff RO, Dosoretz DE, Longgood RM, et al. Meningioma: analysis of recurrence and progression following neurosurgical resection. J Neurosurg 1985;62:18-24.

26.Jnskelninen J, Haltia M, Servo A. Atypical and anaplastic meningiomas. radiology, surgery, radiotherapy and outcome. Surg Neurol 1986;25:233-42.

27.May PL, Broome JC, Lawry J, et al. The prediction of recurrence in meningiomas. A flow cytometric study of paraffin-embedded archival material. J Neurosurg 1989;71:347-51.

28.Douglas H. Haemorrhage in the new-born. Lancet 1966; 1:816-817

29.Mountain KR, Hirsch J, Gallus AS. Neonatal coagulation defect due to anticonvulsant drug treatment in pregnancy. Lancet 1970;1:265-268.

30.Balki M, Manninen PH. Craniotomy for 573 suprasellar meningioma in a 28 -week pregnant woman without fetal heart rate monitoring. Can J Anesth 2004; 51:573-6.

31.Grunberg SM, Weiss MH, Spitz IM et al. Treatment of unresectable meningiomas with the antiprogesterone agent mifepristone. J Neurosurg 1991;74:861-6.

32.Adams RD, Victor M, Ropper AH. Principles ofneurology. 7th ed. New York: Mc Graw-Hill; 2001. 33.Indiyarty $\mathrm{R}$. Occipito-cervical meningioma in pregnancy. Univ Med 2008; 27: 143-9. 